\title{
Development of dual-probe atomic force microscopy system using optical beam deflection sensors with obliquely incident laser beams
}

\section{$\operatorname{AUTHOR}(S)$ :}

Tsunemi, Eika; Kobayashi, Kei; Matsushige, Kazumi; Yamada, Hirofumi

\section{CITATION:}

Tsunemi, Eika ... [et al]. Development of dual-probe atomic force microscopy system using optical beam deflection sensors with obliquely incident laser beams. REVIEW OF SCIENTIFIC INSTRUMENTS 2011, 82(3): 033708.

\section{ISSUE DATE:}

2011-03

URL:

http://hdl.handle.net/2433/160661

\section{RIGHT:}

Copyright 2011 American Institute of Physics. This article may be downloaded for personal use only. Any other use requires prior permission of the author and the American Institute of Physics. The following article appeared in REVIEW OF SCIENTIFIC INSTRUMENTS 82, 033708 (2011) and may be found at http://link.aip.org/link/?rsi/82/033708 


\section{AIP Review of Scientific Instruments}

Development of dual-probe atomic force microscopy system using optical beam deflection sensors with obliquely incident laser beams

Eika Tsunemi, Kei Kobayashi, Kazumi Matsushige, and Hirofumi Yamada

Citation: Rev. Sci. Instrum. 82, 033708 (2011); doi: 10.1063/1.3534830

View online: http://dx.doi.org/10.1063/1.3534830

View Table of Contents: http://rsi.aip.org/resource/1/RSINAK/v82/i3

Published by the American Institute of Physics.

\section{Related Articles}

Stochastic simulation of tip-sample interactions in atomic force microscopy Appl. Phys. Lett. 101, 113105 (2012)

Improving the signal-to-noise ratio of high-speed contact mode atomic force microscopy Rev. Sci. Instrum. 83, 083710 (2012)

Contrast distortion induced by modulation voltage in scanning capacitance microscopy Appl. Phys. Lett. 101, 083503 (2012)

Internal resonance based sensing in non-contact atomic force microscopy Appl. Phys. Lett. 101, 053106 (2012)

Joint strength measurements of individual fiber-fiber bonds: An atomic force microscopy based method Rev. Sci. Instrum. 83, 073902 (2012)

\section{Additional information on Rev. Sci. Instrum.}

Journal Homepage: http://rsi.aip.org

Journal Information: http://rsi.aip.org/about/about_the_journal

Top downloads: http://rsi.aip.org/features/most_downloaded

Information for Authors: http://rsi.aip.org/authors

\section{ADVERTISEMENT}

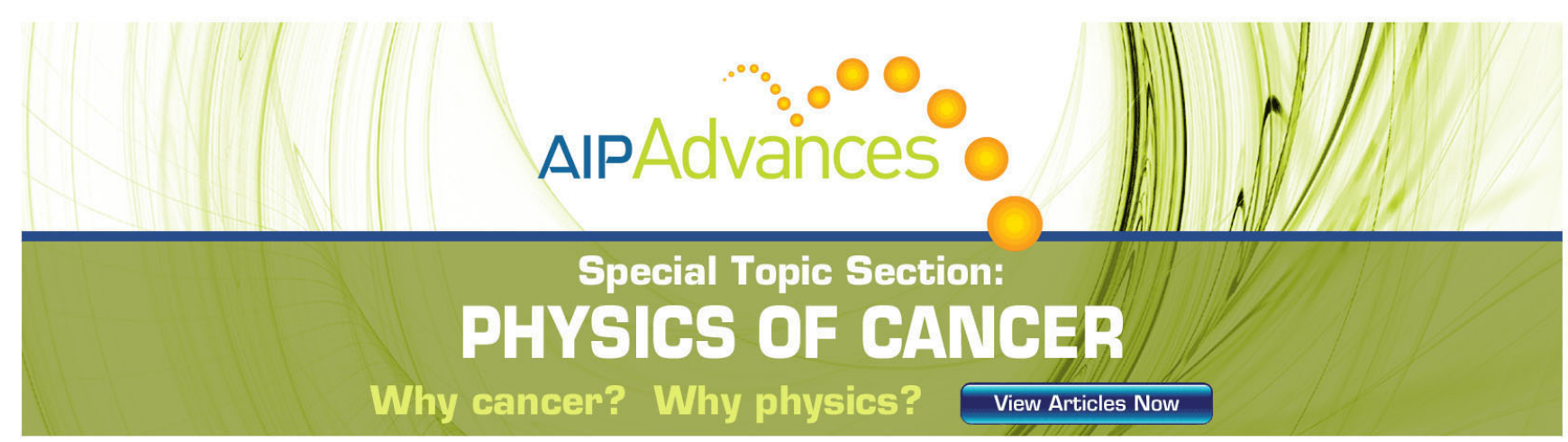




\title{
Development of dual-probe atomic force microscopy system using optical beam deflection sensors with obliquely incident laser beams
}

\author{
Eika Tsunemi, ${ }^{1}$ Kei Kobayashi, ${ }^{2}$ Kazumi Matsushige, ${ }^{1}$ and Hirofumi Yamada ${ }^{1, a)}$ \\ ${ }^{1}$ Department of Electronic Science and Engineering, Kyoto University, Katsura, Nishikyo, Kyoto 615-8510, \\ Japan \\ ${ }^{2}$ Innovative Collaboration Center, Kyoto University, Katsura, Nishikyo, Kyoto 615-8520, Japan
}

(Received 13 July 2010; accepted 11 December 2010; published online 10 March 2011)

\begin{abstract}
We developed a dual-probe (DP) atomic force microscopy (AFM) system that has two independently controlled probes. The deflection of each cantilever is measured by the optical beam deflection (OBD) method. In order to keep a large space over the two probes for an objective lens with a large numerical aperture, we employed the OBD sensors with obliquely incident laser beams. In this paper, we describe the details of our developed DP-AFM system, including analysis of the sensitivity of the OBD sensor for detection of the cantilever deflection. We also describe a method to eliminate the crosstalk caused by the vertical translation of the cantilever. In addition, we demonstrate simultaneous topographic imaging of a test sample by the two probes and surface potential measurement on an $\alpha$-sexithiophene ( $\alpha-6 \mathrm{~T})$ thin film by one probe while electrical charges were injected by the other probe. () 2011 American Institute of Physics. [doi:10.1063/1.3534830]
\end{abstract}

\section{INTRODUCTION}

Atomic force microscopy (AFM), which was developed more than 2 decades ago, has become an indispensable tool in nanotechnology and nanoscience. The AFM is not only used for high-resolution topographic imaging but also for mapping such surface properties as electric, ${ }^{1,2}$ magnetic, ${ }^{3}$ and mechanical ${ }^{4}$ properties. This tool is also used to fabricate ${ }^{5}$ nanostructures and to manipulate atoms ${ }^{6}$ and molecules. ${ }^{7}$ However, the capabilities of AFM systems are often limited because they usually have only one probe. For example, for measuring the in-plane conductance of one-dimensional nanomaterials, an extra electrode must be attached to the sample prior to the measurement. ${ }^{8}$ Several groups showed excellent results on conductance measurements of nanowires by developing multiprobe scanning tunneling microscopy (MP-STM) systems. ${ }^{9-12}$ However, applications of MP-STM, which are limited to samples on conductive substrates, are not applicable to electric measurements on realistic electronic devices that consist of semiconductors and insulators. As an another example, for fabricating nanostructures, since a cantilever suitable for fabrication is not always the best choice for imaging the results at high resolution, investigating the fabricated nanostructures in detail is often difficult. ${ }^{13}$

To overcome such limitations, we developed a dual-probe (DP) AFM system with two independently controlled probes. Since the two probes can be located at any position in an area of concern, various experiments can be performed using the DP-AFM system. We can measure the electrical conductance of nanostructures without attaching an extra electrode to the sample and obtain high-resolution images of an area by probe before and after its modification by the other probe. Moreover, it would be interesting to detect the electrical or mechanical responses of nanostructures or biomolecules to

a)Electronic mail: h-yamada@kuee.kyoto-u.ac.jp. local stimuli being applied by the other probe in real time. We believe that these measurements will accelerate the development of nanosensors, nanoelectronics, and biomimetic devices.

We previously developed a DP-AFM system using such self-sensing cantilevers as piezoresistive or piezoelectric ${ }^{14}$ because they simplify instrumentation. However, applications of the DP-AFM system were limited because only a few types of self-sensing cantilevers are commercially available, and it is difficult to use piezoelectric cantilevers in the contact mode. Therefore, we decided to develop a DP-AFM system that adopts the optical beam deflection (OBD) method $^{15}$ for sensing cantilever deflection, which has generally been used in commercial AFM instruments. We can select suitable cantilevers depending on the applications from a wide variety of commercially available cantilever types. Moreover, the OBD sensor generally has better deflection sensitivity than selfsensing cantilevers. ${ }^{16}$

First, we developed a prototype of the DP-AFM system using the OBD method to test the possible arrangements of the optical components of the two OBD sensors a few years ago. ${ }^{17}$ Since then, we continued developing the DP-AFM system by minimized the optical path in each OBD sensor and improving the mechanical stability and rigidity of each mechanical component. Now the DP-AFM system using the OBD method has been developed, and we recently performed an experiment on the visualization of anisotropic conductance in a polydiacetylene crystal using the system. ${ }^{18}$

In this paper, we review the DP-AFM system with a focus on the OBD sensors with obliquely incident laser beams. We analyze the sensitivity of the OBD sensor for detection of cantilever deflection, and we also propose a method for eliminating crosstalk caused by the vertical translation of the cantilever. Finally, we show several experimental results including simultaneous topographic imaging using the two probes and measurement of the electrical property of an organic thin film. 


\section{OVERVIEW OF SYSTEM}

The DP-AFM system consists of two cantilever probes with three-dimensional positioners, the OBD sensors for each probe, a sample stage with a tube scanner, an optical microscope, and control electronics. Figure 1(a) shows a photograph of a core part of the DP-AFM system. Two probes with positioners, Probe 1 and Probe 2, and the optical components for the OBD sensors, are mounted on a base block as shown in the photograph. An inset in Fig. 1(a) shows an optical micrograph of the two probes located close to each other on a polydiacetylene crystal surface.

In the MP-AFM system, it is essential to control the relative positions of probes on a sample with very high accuracy. Therefore, using of an optical microscope equipped with a large numerical aperture (NA) objective lens is desired. However, a laser diode or a beam splitter cube is generally mounted above the cantilever in conventional OBD sensor to prevent the use of objective lens with a large NA that typically has a short work distance.

We developed the OBD sensors with obliquely incident laser beams for using a decent objective lens so that we have a large space over the probes and the sample. In this setup, the laser beams from semiconductor laser diodes (Hitachi: HL6312G) are brought diagonally downward to the cantilevers and reflected diagonally again to segmented photodiodes (Hamamatsu: S4349), as schematically shown in Fig. 1(a). Figure 1(b) shows a schematic of the OBD sensors with obliquely incident laser beams. Two sets of optical elements were arranged in a mirror symmetric manner. We used an objective lens (Olympus: SLMPlan $50 \times$ ) with an NA of 0.45 and a working distance of $15.0 \mathrm{~mm}$. We used laser drivers and photodiode amplifiers identical to those described in Ref. 19.

Each probe was mounted on a three-dimensional positioner (Unisoku: UMP-1000), which controls the position of the probe. The positioner consists of three inertial sliders using piezostack actuators, each of which translates the probe holder in the $x, y$, or $z$ directions. The probes can either be moved step-by-step by applying ramp pulses to the actuators (stick-slip mode) or statically by applying dc voltages (dc mode). The positions of each probe are controlled using stickslip motion with a step smaller than $500 \mathrm{~nm}$, which is less than the maximum stroke of the sliders in the dc mode (about $2 \mu \mathrm{m}$ for $600 \mathrm{~V}_{\text {peak-to-peak }}$ ). In addition, an extra piezostack is mounted on the probe holder in each positioner, which translates the probe in the $z$ direction. The maximum stroke of the extra piezostack is also about $2.0 \mu \mathrm{m}\left(600 \mathrm{~V}_{\text {peak-to-peak }}\right)$, which is large enough for feedback control of the distance between a probe tip and the sample surface. Therefore, topographic images are independently obtained by each probe. The sample stage is also mounted on a tube scanner that scans the sample in the $x, y$, and $z$ directions. The maximum scan area of the sample is about $20 \mu \mathrm{m} \times 20 \mu \mathrm{m}$ in the $x$ and $y$ directions. The maximum stroke of the tube scanner in the $z$ direction is about $5.0 \mu \mathrm{m}$. The tube scanner is useful for operating the DP-AFM system as a single-probe AFM instrument using one of the two probes and for simultaneously imaging with the two probes. In the latter case, the distance between each probe and the sample surface is controlled by independently translating each probe in the $z$ direction while the sample is scanned in the $x$ and $y$ directions.

A commercial scanning probe microscope (SPM) controller (RHK Technology: SPM-1000) was used for automatic coarse approach, scan signal generation, and image acquisition. We built such additional circuits as self-oscillation electronics for frequency modulation (FM) AFM (Ref. 20) and feedback electronics for controlling the tip-sample distance. Computer software programed by LabVIEW (National Instruments) was used to control the three-dimensional positioners during the coarse positioning of the probes.

The probes and the sample stage were electrically isolated from each other so that we could apply different bias voltages. We can use each probe as a test lead to measure the current versus voltage characteristics between the probes. We can also perform local electrical measurements with each probe using AFM-related techniques, such as electric force microscopy, ${ }^{1,2}$ Kelvin-probe force microscopy (KFM), ${ }^{21}$ and scanning gate microscopy.

\section{OPTICAL BEAM DEFLECTION SENSOR WITH OBLIQUELY INCIDENT LASER BEAM}

In this section, we describe the details of the OBD sensor with obliquely incident laser beams, analyze its sensitivity for detection of cantilever deflection, and propose a method for eliminating the crosstalk caused by the vertical translation of cantilever.

\section{A. Sensitivity of sensor for cantilever deflection and translation}

For simultaneous imaging using the two probes, we control the positions of each probe in the $z$ direction to maintain a constant distance between the tip and the sample surface while we scan the sample in parallel to the sample surface. The AFM instruments in which the probe is translated during a scan ${ }^{22-24}$ are often designed to achieve a large scan area without large distortion caused by a tube scanner. It is often argued that cantilever translation causes crosstalk in the deflection signal in such AFM instruments. Therefore, the influence of the translation of the probe must be considered relative to the OBD sensor elements. Here we analyze the sensitivities of the OBD sensor for the cantilever's deflection and translation and the crosstalk of the deflection and the translation.

We consider a semisphere with a radius of $r$ in a spherical coordinate system $(r, \theta, \varphi)$ surrounding the cantilever, where $0<\theta<\pi$ and $0<\varphi<\pi$ [Fig. 2(a)]. The bottom plane of the semisphere includes the cantilever plane, and the $x$ axis is parallel to the long axis of the cantilever. We assume that a collimated laser beam comes in through point $\mathrm{A}\left(r, \theta_{\mathrm{a}}, \varphi_{\mathrm{a}}\right)$ on the semisphere surface and is reflected at origin $(0,0,0)$. Then the laser spot on a segmented photodiode located at point $\mathrm{B}\left(r, \theta_{\mathrm{b}}, \varphi_{\mathrm{b}}\right)$ is detected, which is on the semisphere surface for simplicity. Therefore, $r$ corresponds to the distance between the cantilever and the photodiode, $l_{\mathrm{PD}}$. We also assume that the active surface of the photodiode is adjusted perpendicular to the reflected laser beam. 
(a)

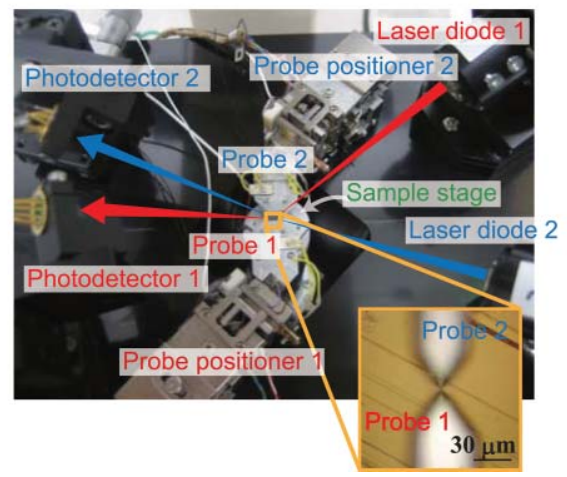

(b)

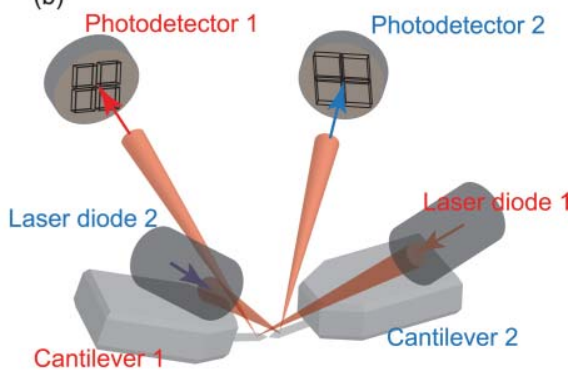

FIG. 1. (Color online) (a) Photograph of a core part of the DP-AFM system. An optical micrograph of the two probes and a sample surface is also shown as an inset. (b) Schematic of the optical beam deflection sensors with obliquely incident laser beams.

First, we consider the displacement of the laser spot on the photodiode caused by the cantilever deflection. The relationship between the coordinates of points A and B is written as

$$
\left\{\begin{array}{l}
\theta_{\mathrm{b}}=\pi-\theta_{\mathrm{a}} \\
\varphi_{\mathrm{b}}=\varphi_{\mathrm{a}}
\end{array}\right.
$$

The cantilever tilt with respect to the bottom plane of the semisphere by angle $\Delta \alpha$ is equivalent to the rotation of the coordinate system in the $x-z$ plane angle $-\Delta \alpha$. Therefore, the position of point $\mathrm{B}$ is shifted by $-2 \Delta \alpha$. Since the Cartesian coordinates of point $\mathrm{B}$ are $\left(-r \cos \theta_{\mathrm{a}} \cos \varphi_{\mathrm{a}}, r \cos \theta_{\mathrm{a}} \sin \varphi_{\mathrm{a}}, r \sin \theta_{\mathrm{a}}\right)$, the displacement of the laser spot on segmented photodiode $\left(D_{\alpha}\right)$ caused by cantilever deflection $d_{\alpha}$ becomes

$$
\begin{aligned}
D_{\alpha} & =2 \Delta \alpha \sqrt{r^{2}-\left(r \cos \theta_{\mathrm{a}} \sin \varphi_{\mathrm{a}}\right)^{2}} \\
& =2 r \Delta \alpha \sqrt{1-\cos ^{2} \theta_{\mathrm{a}} \sin ^{2} \varphi_{\mathrm{a}} .}
\end{aligned}
$$

The tilt angle of the cantilever $\Delta \alpha$ caused by the cantilever deflection $d_{\alpha}$ is described as $\Delta \alpha=(3 / 2)\left(d_{\alpha} / l_{\mathrm{c}}\right)$, where $l_{\mathrm{c}}$ is the length of the cantilever. ${ }^{25}$ Therefore, Eq. (2) becomes

$$
D_{\alpha}=3 r\left(d_{\alpha} / l_{\mathrm{c}}\right) \sqrt{1-\cos ^{2} \theta_{\mathrm{a}} \sin ^{2} \varphi_{\mathrm{a}}} .
$$

Note that these equations hold for the conventional OBD sensor with vertically incident laser beam $\left(\varphi_{\mathrm{a}}=0\right)$.

Next we consider the displacement of the laser spot caused by the cantilever translation in a direction perpendicular to the sample surface. If we assume that the cantilever is translated by $\Delta z$ in the $z$ direction in Fig. 2(a), the displacement of the laser spot on photodiode $\left(D_{\perp}\right)$ can be calculated from a cross-sectional schematic of the semisphere in the $r-\theta$ plane Fig. 2(b) as

$$
D_{\perp}=2 \Delta z \cos \theta_{\mathrm{a}} .
$$

The cantilever is generally mounted on the cantilever holder with small tilt angle $\alpha$ with respect to the sample surface. Therefore, the translation of the cantilever $d_{\perp}$ in the direction perpendicular to the sample surface corresponds to translation $\Delta z$ in the $z$ direction as

$$
\Delta z=d_{\perp} \cos \alpha
$$

and we obtain

$$
D_{\perp}=2 d_{\perp} \cos \alpha \cos \theta_{\mathrm{a}}
$$

Finally, we calculate the ratio between signals $S_{\alpha}$ and $S_{\perp}$ for the deflection and translation. If we assume that the output signal of the photodiode is proportional to the displacement of the laser spot on the photodiode, we obtain

$$
\frac{S_{\alpha}}{S_{\perp}}=\frac{D_{\alpha}}{D_{\perp}}=\frac{3 r \sqrt{1-\cos ^{2} \theta_{\mathrm{a}} \sin ^{2} \varphi_{\mathrm{a}}}}{2 l_{\mathrm{c}} \cos \alpha \cos \theta_{\mathrm{a}}}
$$

by setting $d_{\alpha}=d_{\perp}$.

\section{B. Separation of translation signal from deflection signal}

As discussed in Sec. III A., the cantilever translation in the $z$ direction also causes the displacement of the laser spot on the photodiode, which causes crosstalk in the deflection signal. In the OBD sensors with a vertically incident laser beam, the angle between $r-\theta$ and $x-z$ planes, $\varphi$, is zero. Therefore, the directions of the displacement of the laser spot on the photodiode caused by the deflection and translation are indistinguishable. However, in the OBD sensors with obliquely incident laser beams, the direction of the displacement caused by the vertical translation is not the same as that caused by the deflection. The crosstalk can be eliminated as follows.
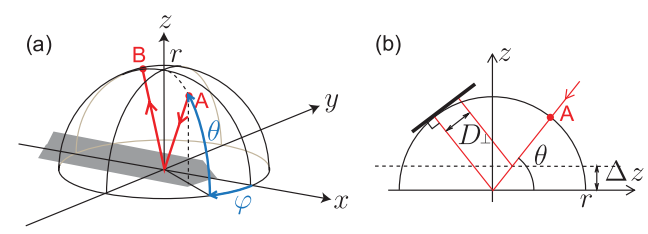

FIG. 2. (Color online) (a) Schematic of a semisphere system for calculating the sensitivities of the OBD sensor to the cantilever deflection and translation. (b) Cross-sectional schematic of the semisphere showing the relation between the cantilever translation in the $z$ direction and the displacement of the laser spot on the segmented photodiode. 
(a)

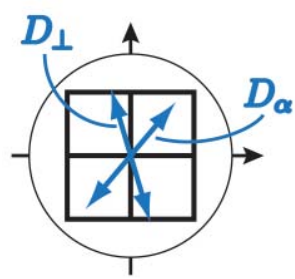

(b)

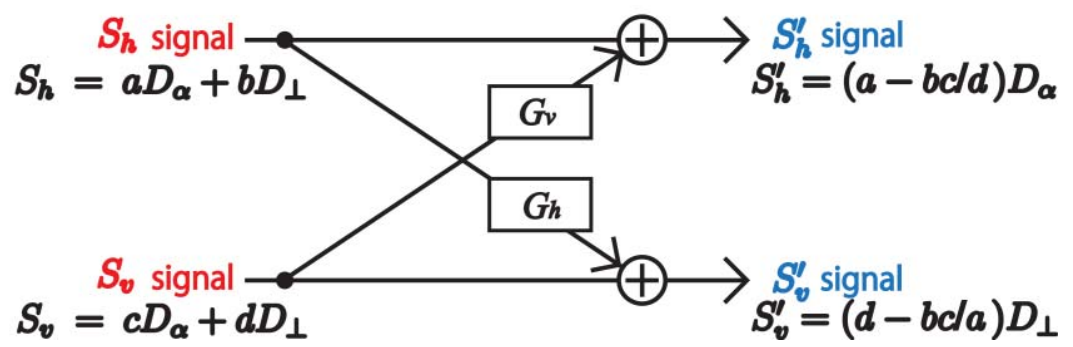

FIG. 3. (Color online) (a) Schematic of a segmented photodiode showing the displacement $D_{\alpha}$ and $D_{\perp}$. They are not generally perpendicular. (b) Schematic of a procedure to eliminate the crosstalk between the deflection and translation signals.

The OBD signals caused by deflection $d_{\alpha}$ and translation $d_{\perp}$ cause the displacement of the spot on the photodiode, $D_{\alpha}$ and $D_{\perp}$, respectively. Two signals, $S_{h}$ and $S_{v}$, corresponding to the horizontal and vertical displacements on the segmented photodiode, are obtained as shown in Fig. 3(a),

$$
\left\{\begin{array}{l}
S_{h}=a D_{\alpha}+b D_{\perp}, \\
S_{v}=c D_{\alpha}+d D_{\perp},
\end{array}\right.
$$

where $a, b, c$, and $d$ are constants corresponding to the sensitivities of the deflection and translation, which are dependent on the geometry of the cantilever and the arrangement of the optical elements and the laser power.

If we mount the photodiode so that its vertical axis is parallel to the direction of $D_{\perp}, b$ becomes zero and $S_{h}$ is not affected by the cantilever translation. However, the deflection sensitivity is not optimized in this arrangement. Moreover, since the axis is not exactly adjusted parallel to the direction of $D_{\perp}, S_{h}$ is indeed affected by the cantilever translation.

To obtain maximum sensitivity for deflection $d_{\alpha}$, we mounted the photodiode so that its horizontal axis is roughly parallel to the direction of $D_{\alpha}$. Then we built a simple circuit to eliminate $D_{\perp}$ and $D_{\alpha}$ components from the $S_{h}$ and $S_{v}$ signals, respectively, as shown in Fig. 3(b). We can easily tune the gains of variable gain amplifiers $G_{v}$ and $G_{h}$ to $-(b / d)$ and $-(c / a)$, respectively, to obtain two separated signals, as described in Sec. III C. We can use the horizontal signal after correction $\left(S_{h}^{\prime}\right)$ for regulating the tip-sample distance during AFM imaging without being influenced by the cantilever translation $d_{\perp}$.

\section{Performance of optical beam deflection sensor}

We measured the sensitivity of the OBD sensor to the cantilever deflection in the DP-AFM system. The parameters of the optical arrangement are $r=50 \mathrm{~mm}$, $\theta=31^{\circ}, \varphi=66^{\circ}$, and $\alpha=13^{\circ}$. Figure 4 is the thermal noise spectrum of a cantilever with a nominal spring constant $(k)$ of $42 \mathrm{~N} / \mathrm{m}$ (NanoWorld AG: NCHR), measured using the OBD sensor with an obliquely incident laser beam. The laser power received at the photodiode was about $2 \mathrm{~mW}$. In this measurement, we measured the spectrum of the $S_{h}$ signal before correction. The noise-equivalent deflection density $\left(N_{\mathrm{ds}}\right)$ was calculated as $34 \mathrm{fm} / \sqrt{\mathrm{Hz}}$, by fitting the measured spectrum to the theoretical curve for the total noise-equivalent deflection density
$\left(N_{\text {total }}\right)$, which is written as

$$
N_{\text {total }}=\sqrt{N_{\mathrm{ds}}^{2}+\frac{\omega_{0}^{4}}{\left(\omega^{2}-\omega_{0}^{2}\right)^{2}+\omega^{2} \omega_{0}^{2} / Q^{2}} \frac{4 k_{\mathrm{B}} T}{k \omega_{0} Q}},
$$

where $N_{\text {total }}, \omega_{0}, Q, k_{\mathrm{B}}$, and $T$ are the total noise-equivalent deflection density, the angular resonance frequency and Q-factor of the cantilever, the Boltzmann constant, and temperature. ${ }^{26}$ We also detected $S_{h}$ and $S_{v}$ signals while translating a probe toward a silicon substrate by applying a ramp wave to the shear piezostack for movement in the $z$ direction of the probe to calculate the sensitivity of the OBD sensor to the cantilever deflection and translation. The sensitivity of the OBD sensor to the cantilever deflection was calculated as $\sqrt{\left.S_{h(\alpha)}{ }^{2}+S_{v(\alpha)}\right)^{2}} / d_{\alpha} . S_{h(\alpha)} / d_{\alpha}$ and $S_{v(\alpha)} / d_{\alpha}$ are the horizontal and vertical signals normalized to a unit deflection, respectively, which were measured by translating the probe in contact with the cantilever tip. We also measured the sensitivity of the OBD sensor to the cantilever translation, $\sqrt{S_{h(z)}^{2}+S_{v(z)}^{2}} / d_{\perp} . S_{h(z)} / d_{\perp}$ and $S_{v(z)} / d_{\perp}$ are the horizontal and vertical signals normalized to a unit translation, respectively, which were measured by translating the cantilever in the $z$ direction above the sample.

The ratio of the measured sensitivity for the cantilever deflection to that for the translation, $S_{\alpha} / S_{\perp}$, was 159 . This measured value was smaller compared to the value calculated using Eq. (7), which was 348. There are several possible reasons for the difference. The diameter of the focused laser spot of the oblique incidence laser was about $50 \mu \mathrm{m}$ or more, which is slightly larger than the cantilever width. Therefore, the laser

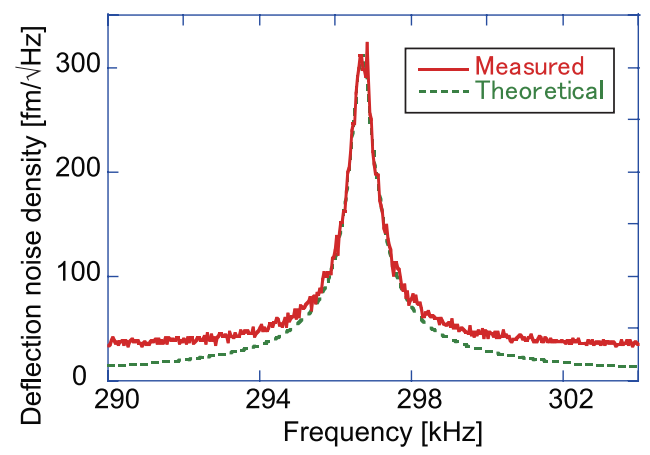

FIG. 4. (Color online) Frequency spectrum of $S_{h}$ signal from the photodiode showing the thermal noise spectrum of a cantilever (NCHR) in the DP-AFM system. A theoretical fitting curve [Eq. (9)] is also shown. 

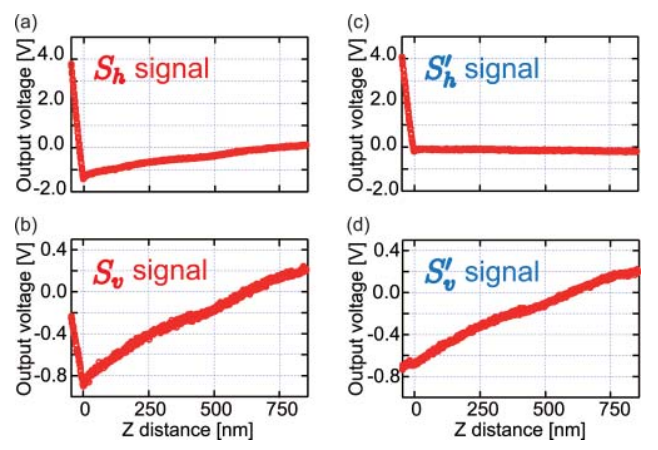

FIG. 5. (Color online) (a) and (b) Horizontal $\left(S_{h}\right)$ and vertical $\left(S_{v}\right)$ signals from the segmented photodiode recorded during translation of a cantilever vertically toward a silicon substrate. (c) and (d) Corrected horizontal $\left(S_{h}^{\prime}\right)$ and vertical $\left(S_{v}^{\prime}\right)$ signals obtained through a compensation circuit recorded simultaneously with (a) and (b). The origin of the distance axis was defined as the point of contact.

spot on the photodiode shows an irregular and anisotropic diffraction pattern, which makes us difficult to precisely calculate the sensitivity. Also due to the large spot size on the cantilever, the spot was not exactly at the end of the cantilever as it is in the assumption for the calculation. The total laser power received at the PD was also slightly changed during the measurement up to $5 \%$ in the worst case.

In the following paragraph, we demonstrate the compensation of the crosstalk caused by the cantilever translation. The photodiode was mounted so that its horizontal axis was roughly parallel to the direction of $D_{\alpha}$. We used a cantilever (NanoWorld AG: ATEC-NC) with parameters $l_{\mathrm{c}}=160 \mu \mathrm{m}$ and $k=45 \mathrm{~N} / \mathrm{m}$. We fed the $S_{h}$ and $S_{v}$ signals into the compensation circuit, then we modulated either $D_{\alpha}$ and $D_{\perp}$ on the OBD sensor independently by applying ac signals to the piezoelectric plate for cantilever excitation and the additional $z$ shear piezostack. $G_{h}$ was tuned to minimize the ac component in the $S_{v}^{\prime}$ signal while the cantilever was oscillated, and $G_{v}$ was tuned to minimize the ac component in the $S_{h}^{\prime}$ signal while the cantilever was translated sinusoidally along the direction perpendicular to the sample surface without cantilever oscillation.

We recorded the $S_{h}, S_{v}$ signals and the corrected signals, $S_{h}^{\prime}$ and $S_{v}^{\prime}$, while vertically translating the cantilever toward a silicon substrate as shown in Fig. 5. Crosstalk was observed in $S_{h}$ and $S_{v}$. Both $S_{h}$ and $S_{v}$ signals were gradually changed while the distance was in the range from 0 to $850 \mathrm{~nm}$. Then both signals showed abrupt changes at the contact point. On the other hand, the influence of the cantilever translation was almost eliminated in the $S_{h}^{\prime}$ signal. In addition, the $S_{v}^{\prime}$ signal did not show an abrupt change at the contact point. It should be mentioned that since the $S_{v}^{\prime}$ signal represents the cantilever translation and is not influenced by the cantilever deflection, the signal can be used to compensate the nonlinearity of the piezostack.

\section{EXPERIMENTAL RESULTS}

\section{A. Simultaneous AFM imaging}

To bring the two probes to their target positions in the DP-AFM system, it is essential to simultaneously obtain topo-

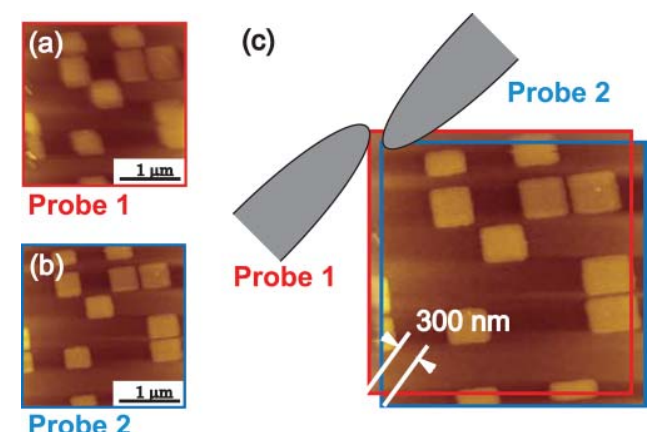

FIG. 6. (Color online) FM-AFM images of an address-patterned sample simultaneously obtained using Probe 1 (a) and Probe 2 (b). (c) Overlapped image of (a) and (b) indicating that the distance between the two tips was about $300 \mathrm{~nm}$. The rest positions of the tips are also schematically shown with their pointing directions.

graphic images with the two probes and to recognize their positions on the sample surface. In this section, the procedure for determining the relative positions of the probes is described. We used two silicon cantilevers (NanoWorld AG: ATEC-NC) with tetrahedral tips that slightly protrude at an angle from the cantilever end. We can observe the tip from the top with an optical microscope even when the cantilevers are mounted with a slight tilt angle $\alpha$. The nominal spring constant and the resonance frequency were $45 \mathrm{~N} / \mathrm{m}$ and $335 \mathrm{kHz}$. The sample used in this experiment was 10 -nm-thick platinum patterned islands on a silicon oxide surface. Pt islands form an array of binary patterns that represent their addresses and show their absolute positions on the substrate. The cantilevers were excited at their resonance frequencies with a self-oscillation loop, and we regulated the tip-sample distance by keeping the oscillation frequency constant (FM-AFM).

First, we brought the two probes close to each other with the optical microscope until the distance between the probes became a few micrometers. Then we adjusted the direction of the laser beams and the positions of the segmented photodiodes. The probes were brought close to the surface, and we obtained two AFM images by scanning the sample while the distance between each probe and the sample surface was independently controlled. When the two probes were close enough, we found overlapping features between the two images, which indicate the relative positions of the two probe tips. After identifying the relative positions of the probes, we translated the probes to shorten the distance between the tips. We repeated these procedures until the two probes were located on their target locations. Figures 6(a) and 6(b) are the AFM images obtained simultaneously using Probe 1 and Probe 2 after repeating the above procedures. From the overlap of the features in the two images, the distance between the two tips can be measured as about $300 \mathrm{~nm}$, as shown in Fig. 6(c). We also schematically indicated the rest positions of the tips by their pointing directions. With these procedures, we can routinely make the distance between the two probe tips short to a few hundred nanometer and bring each probe to the target position on any sample surface. Note that it was difficult to bring the two tips within a distance of $1 \mu \mathrm{m}$ was difficult in the prototype system. ${ }^{17}$ 


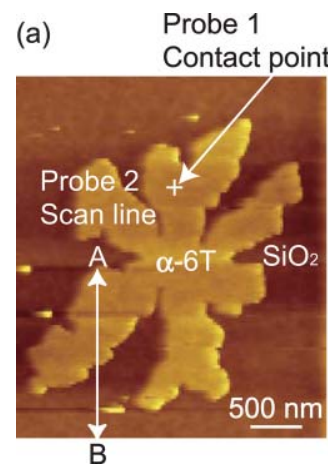

(b)

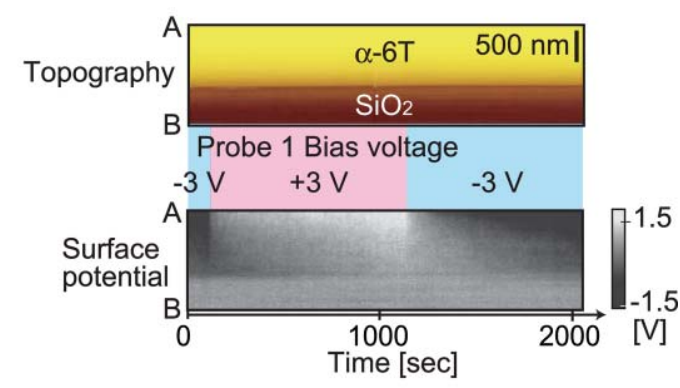

FIG. 7. (Color online) (a) FM-AFM image of a dendric island of $\alpha-6 \mathrm{~T}$ thin film. (b) Images showing time evolution of the surface potential profile (bottom) and the topographic profile (top) along the A-B line indicated in (a).

\section{B. Electrical measurement on organic thin film}

We also performed local electrical measurements of an organic semiconductor thin film with the DP-AFM system. Recently, organic semiconductors, which have been attracting great attention as functional materials, have been widely studied. We can measure the local electrical properties of organic semiconductors with the DP-AFM system, which might provide insights into the relation between the local structures and the electrical characteristics. As a preliminary study, we injected charges into an organic semiconductor thin film island on a silicon oxide surface with one probe and simultaneously measured the surface potential of the island with the other probe at the same time.

We deposited $\alpha-6 \mathrm{~T}$ films on a high-doped Si substrate with a $300 \mathrm{~nm}$-thick $\mathrm{SiO}_{2}$ layer. A PtIr-coated conductive cantilever with a high spring constant (NanoWorld AG: ATECNCPT, $k=45 \mathrm{~N} / \mathrm{m}$ ) was used as a probe for the KFM measurement and one with a lower spring constant (NanoWorld AG: ATEC-EFM, $k=2.8 \mathrm{~N} / \mathrm{m}$ ) was used as the other probe for contact. The tip-sample distance was regulated by the FM detection method. Figure 7(a) shows an AFM image of a dendritic island of $\alpha$-6T obtained using Probe 2. The height of the film was about $2.0 \mathrm{~nm}$, which indicates the film was the monolayer film. We applied a bias voltage to the island at the position indicated in Fig. 7(a) with Probe 1 while the $\mathrm{Si}$ substrate was electrically grounded. A modulation bias voltage ( $\left.1 \mathrm{kHz}, 2 \mathrm{~V}_{\text {peak-to-peak }}\right)$ was applied to Probe 2, and the dc bias voltage applied to Probe 2 was controlled to perform the KFM measurement. ${ }^{27}$

Figure 7(b) shows the time evolution of the surface potential profiles along the A-B line in Fig. 7(a) while the electrical charges were injected to the island from Probe 1 . We continuously scanned Probe 2 along the A-B line and recorded the topographic and the surface potential profiles along it. The top and bottom images in Fig. 7(b) show the time evolution of the topographic and the surface potential profiles of the film and $\mathrm{SiO}_{2}$. The gradual change of the surface potential on the $\alpha-6 \mathrm{~T}$ island after the application of the bias voltage to Probe 1 $(-3 \mathrm{~V} \rightarrow+3 \mathrm{~V} \rightarrow-3 \mathrm{~V})$ was observed while it was not observed on $\mathrm{SiO}_{2}$. This result indicates the slow diffusion of the injected charges in the $\alpha-6 \mathrm{~T}$ island. The contrasts in the bottom image in Fig. 7(b) indicate that the charges diffused from the area near the contact point of Probe 1 to the surrounding areas.

\section{CONCLUSIONS}

We developed a DP-AFM system with two independently controlled probes. The OBD sensors with obliquely incident laser beams were employed, in which the laser beams were brought diagonally down to the cantilever and reflected diagonally to the segmented photodiodes so that an objective lens with a large NA could be used. We analyzed the sensitivities of the OBD sensor to the deflection and translation of the cantilever. Then we proposed a method to eliminate crosstalk caused by the cantilever translation. We demonstrated simultaneous AFM imaging using the two probes when the distance between the two probe tips was about $300 \mathrm{~nm}$. We also measured the time evolution of the surface potential of $\alpha-6 \mathrm{~T}$ monolayer island by FM-KFM while the electrical charges were locally injected into the island.

\section{ACKNOWLEDGMENTS}

This work was supported by Grants-in-Aid for Scientific Research from the Ministry of Education, Culture, Sports, Science and Technology of Japan. We also acknowledge the Research Fellowships for Young Scientists Program and the Global COE Program of the Japanese Society for the Promotion of Science.

${ }^{1}$ J. E. Stern, B. D. Terris, H. J. Mamin, and D. Rugar, Appl. Phys. Lett. 53, 2717 (1988).

${ }^{2}$ B. D. Terris, J. E. Stern, D. Rugar, and H. J. Mamin, J. Vac. Sci. Technol. A 8, 374 (1990).

${ }^{3}$ Y. Martin and H. K. Wickramasinghe, Appl. Phys. Lett. 50, 1455 (1987).

${ }^{4}$ P. Maivald, H. J. Butt, S. A. C. Gould, C. B. Prater, B. Drake, J. A. Gurley,

V. B. Elings, and P. K. Hansma, Nanotechnology 2, 103 (1991).

${ }^{5}$ E. S. Snow and P. M. Campbell, Science 270, 1639 (1995).

${ }^{6}$ Y. Sugimoto, P. Jelinek, P. Pou, M. Abe, S. Morita, R. Perez, and O. Custance, Phys. Rev. Lett. 98, 106104 (2007).

${ }^{7}$ A. L. Weisenhorn, J. E. M. Dougall, S. A. C. Gould, S. D. Cox, W. S. Wise, J. Massie, P. Maivald, V. B. Elings, G. D. Stucky, and P. K. Hansma, Science 247, 1330 (1990).

${ }^{8}$ T. Yajima, H. Tanaka, T. Matsumoto, Y. Otsuka, Y. Sugawara, and T. Ogawa, Nanotechnology 18, 095501 (2007).

${ }^{9}$ S. Tsukamoto, B. Siu, and N. Nakagiri, Rev. Sci. Instrum. 62, 1767 (1991).

${ }^{10}$ O. Kubo, Y. Shingaya, M. Nakaya, M. Aono, and T. Nakayama, Appl. Phys. Lett. 88, 254101 (2006).

${ }^{11}$ X. Lin, X. B. He, T. Z. Yang, W. Guo, D. X. Shi, H.-J. Gao, D. D. D. Ma, S. T. Lee, F. Liu, and X. C. Xie, Appl. Phys. Lett. 89, 043103 (2006).

${ }^{12} \mathrm{~S}$. Yoshimoto, Y. Murata, K. Kubo, K. Tomita, K. Motoyoshi, T. Kimura, H. Okino, R. Hobara, I. Matsuda, S. Honda, M. Katayama, and S. Hasegawa, Nano Lett. 7, 956 (2007). 
${ }^{13}$ S. Ohkouchi, Y. Nakamura, H. Nakamura, and M. Asakawa, Jpn. J. Appl. Phys., Part 1 44, 5777 (2005).

${ }^{14}$ N. Satoh, E. Tsunemi, Y. Miyato, K. Kobayashi, S. Watanabe, T. Fujii, K. Matsushige, and H. Yamada, Jpn. J. Appl. Phys. 46, 5543 (2007).

${ }^{15}$ P. K. Hansma, B. Drake, D. Grigg, C. B. Prater, F. Yashar, G. Gurley, V. Elings, S. Feinstein, and R. Lal, J. Appl. Phys. 76, 796 (1994).

${ }^{16} \mathrm{~S}$. Watanabe and T. Fujii, Rev. Sci. Instrum. 67, 3898 (1996).

${ }^{17}$ E. Tsunemi, N. Satoh, Y. Miyato, K. Kobayashi, K. Matsushige, and H. Yamada, Jpn. J. Appl. Phys. 46, 5636 (2007).

${ }^{18}$ E. Tsunemi, K. Kobayashi, K. Matsushige, and H. Yamada, J. Vac. Sci. Technol. B 28, C4D24 (2010).

${ }^{19}$ T. Fukuma, M. Kimura, K. Kobayashi, K. Matsushige, and H. Yamada, Rev. Sci. Instrum. 76, 053704 (2005).
${ }^{20}$ K. Kobayashi, H. Yamada, H. Itoh, T. Horiuchi, and K. Matsushige, Rev. Sci. Instrum. 72, 4383 (2001).

${ }^{21}$ M. Nonnenmacher, M. P. O'Boyle, and H. K. Wickramasinghe, Appl. Phys. Lett. 58, 2921 (1991).

${ }^{22}$ J. Kwon, J. Hong, Y.-S. Kim, D.-Y. Lee, K. Lee, S. Lee, and S. Park, Rev. Sci. Instrum. 74, 4378 (2003).

${ }^{23}$ K. O. van der Werf, C. A. J. Putman, B. G. de Grooth, F. B. Segerink, E. H. Schipper, N. F. van Hulst, and J. Greve, Rev. Sci. Instrum. 64, 2892 (1993).

${ }^{24}$ D. R. Baselt and J. D. Baldeschwieler, Rev. Sci. Instrum. 64, 908 (1993).

${ }^{25}$ G. Meyer and N. M. Amer, Appl. Phys. Lett. 57, 2089 (1990).

${ }^{26}$ J. E. Sader, J. W. M. Chon, and P. Mulvaney, Rev. Sci. Instrum. 70, 3967 (1999).

${ }^{27}$ S. Kitamura and M. Iwatsuki, Appl. Phys. Lett. 72, 3154 (1998). 